\title{
Impact Analysis of Variable Generation on Small Signal Stability
}

\author{
Jae Woong Shim *†, Student Member IEEE, Gregor Verbič *, Senior Member IEEE, \\ Kyeon Hur ${ }^{\dagger}$, Senior Memebr IEEE, David J. Hill ${ }^{* \ddagger}$, Fellow IEEE \\ ${ }^{*}$ The University of Sydney, Sydney, Australia, \{jae-woong.shim, gregor.verbic, david.hill $\} @$ sydney.edu.au \\ ${ }^{\dagger}$ Yonsei University, Seoul, S. Korea, \{jeuhnshim, khur\}@yonsei.ac.kr \\ $\ddagger$ The University of Hong Kong, Hong Kong, dhill@eee.hku.hk
}

\begin{abstract}
This paper aims to analyse the influence of fluctuating renewables on small-signal stability. Most of the research on renewable energy integration assumes that power is provided from a static generation unit and studies how much damping ratio and frequency changes as a result of changing power and inertia. This research is motivated by mode coupling where one oscillatory mode may have an effect on other modes if the mode frequencies are similar. The paper discusses the impact of fluctuating power sources, Type IV wind turbines in our case, on possible coupling between the fluctuating wind power and the existing modes in the system. Since the fluctuating power may combine a large band of frequency components, the power system can react to any specific frequency of a variable generator. In this paper, several influential frequencies were injected through the renewable generator. System identification is used first to obtain the linearized state-space model of the system and the relevant transfer functions, which are then used to identify possible resonant frequencies. DIgSILENT/Power Factory is used next to analyse the system's response to specific frequencies in the time domain.
\end{abstract}

Index Terms-power system oscillations, small signal stability, mode coupling, renewable energy sources, wind power, resonance.

\section{INTRODUCTION}

$\mathbf{P}$ ENETRATION of intermittent renewable energy sources (RES) is steadily increasing following the renewable portfolio targets that have been put in place in many countries around the world to speed up the transition to more RES based power systems. The University of Melbourne Energy Research Institute's study [1] suggests that a 100\% renewable scenario can be achieved as early as 2020, although the Australian Energy Market Operator (AEMO) considers the 2030 to 2050 time frame to be more realistic for Australia [2]. To tackle the integration of RES, the analysis of grid characteristics with RES is essential, and used for operation and planning in the long-term future. Without the fundamental work for implications of variable generation, future power system may have difficulty operating stably with high penetration of RES.

Much research effort has been put into studying the impact of RES, mostly solar and wind, on power system stability, focusing predominantly on small-signal [3]-[7] and transient [7], [8] angle stability. Such initiatives internationally notwithstanding, the influence of RES on power system stability and performance still requires a lot of attention. The common denominator of the existing research is the use of quasi- static analysis, implicitly assuming that variations in the RES' output are slow enough not to impact the system modes. This approach seems to be widely accepted. For example, a comprehensive CIGRE Technical Brochure [9] and a more recent AEMO study [10] don't even mention this assumption. We make an attempt to cover this gap by considering the output of a RES not to be static at the studied operating point but rather consisting of a fixed component and a superimposed fluctuating component of a frequency similar to the existing system's modes.

The problem of mode coupling hasn't attracted much attention in the power engineering community so far. The earliest references date back to 1980s [11]. The phenomenon has been observed in practice, e.g. in the WECC with implications for PSS tuning [12]. To the best of our knowledge, this is the first attempt to study the impact of RES on mode coupling. The goal of this paper is thus to establish hypotheses about the influence on system stability from variable generators. Unlike the conventional thermal and hydro power plants whose output variation is very slow with no or negligible higherfrequency components, the output of intermittent RES consists of fluctuating components with frequencies that can potentially interact with the existing system modes. This can change the behaviour of the system or even cause instability in a large scale network. The focal point of our discussion is on the influence of the fluctuating frequency component on the small disturbance stability. We first use sub-space system identification to obtain a reduced-order linearized model of the system and the relevant transfer functions. Then we use time-domain simulation to analyse the behaviour of the system subject to penetration of fluctuating RES in the selected frequency bands.

The rest of paper is organized as follows: an introduction discussing the concept of mode coupling and power system oscillations is given in Section II. In Section III, system identification used in the paper is briefly discussed. In Section IV, the results of the case study on test system are given, and Section V concludes the paper.

\section{BACKGROUND}

The main focus of this study is small-signal stability, which is defined as the ability of the power network to keep synchronism under small disturbances, typically changes in 
either load or generation [13]. The changes are considered to be small enough so the system can be linearized around the operating point. The remainder of this section discusses smallsignal stability and mode coupling, which lays the groundwork for the case studies considered later in the paper.

\section{A. Small-signal Stability and Mode Coupling}

The behaviour of a power system can be described by a set of differential and algebraic equations of the form:

$$
\begin{aligned}
\dot{\mathbf{x}} & =\mathbf{f}_{1}\left(\mathbf{x}_{1}, \mathbf{x}_{2}, \mathbf{u}\right) \\
0 & =\mathbf{f}_{2}\left(\mathbf{x}_{1}, \mathbf{x}_{2}, \mathbf{u}\right) \\
\mathbf{y} & =\mathbf{g}_{0}\left(\mathbf{x}_{1}, \mathbf{x}_{2}, \mathbf{u}\right)
\end{aligned}
$$

where $f_{1}, f_{2}$ and $g_{0}$ give the vectors of non-linear differential, algebraic and output equations, respectively; and $\mathbf{x}_{1} \in \mathbb{R}^{n}$, $\mathbf{x}_{2} \in \mathbb{R}^{l}, \mathbf{u} \in \mathbb{R}^{m}$ and $\mathbf{y} \in \mathbb{R}^{p}$ denote the vectors of state variables, algebraic variables, inputs and outputs, respectively. Typically, the system in (1) is assumed time-invariant so the time-derivatives of the state variables are not explicit functions of the time. Assuming $\frac{\partial \mathbf{f}_{2}}{\partial \mathbf{x}_{2}}$ is nonsingular we can eliminate $\mathbf{x}_{2}$. Let $\mathbf{x}=\mathbf{x}_{1}$ and we get

$$
\begin{aligned}
& \dot{\mathbf{x}}=\mathbf{f}(\mathbf{x}, \mathbf{u}) \\
& \mathbf{y}=\mathbf{g}(\mathbf{x}, \mathbf{u})
\end{aligned}
$$

In small-signal stability analysis, the disturbances are considered small so the system can be linearized around the operating point $\left(\mathbf{x}_{0}, \mathbf{u}_{0}\right)$ resulting in

$$
\begin{aligned}
& \Delta \dot{\mathbf{x}}=\left.\frac{\partial \mathbf{f}}{\partial \mathbf{x}}\right|_{0} \Delta \mathbf{x}+\left.\frac{\partial \mathbf{f}}{\partial \mathbf{u}}\right|_{0} \Delta \mathbf{u}=\mathbf{A} \Delta \mathbf{x}+\mathbf{B} \Delta \mathbf{u} \\
& \Delta \mathbf{y}=\left.\frac{\partial \mathbf{f}}{\partial \mathbf{x}}\right|_{0} \Delta \mathbf{x}+\left.\frac{\partial \mathbf{f}}{\partial \mathbf{u}}\right|_{0} \Delta \mathbf{u}=\mathbf{C} \Delta \mathbf{x}+\mathbf{D} \Delta \mathbf{u}
\end{aligned}
$$

where $\Delta \mathbf{x}, \Delta \mathbf{y}$ and $\Delta \mathbf{u}$ represent deviations from operating point values.

The linearized system $(6,7)$ can be used to investigate the system's response to small variations in the input or state variables. A possible instability can be due to lack of synchronizing torque, resulting in an increase in rotor angle through a non-oscillatory or aperiodic mode; or due to lack of sufficient damping torque, resulting in oscillations of increasing amplitude. The oscillations can be either local or global, depending on the machines involved. Global or interarea mode oscillations, involve one group of generators in one part of the system swinging against a group of generators in another part of the system. Local mode oscillations, on the other hand, are restricted to a small part of the system and often refer to one generator's motion with respect to the rest of the system.

Mode coupling is a situation where an oscillation mode in one part of the system interacts with a mode of oscillation in a remote part [11]. Mode coupling had been observed in the WECC when studying the placement of PSSs [12]. It was found that a generator in one part of the system had a relatively high participating factor in an oscillatory mode associated with a group of generators located in a remote part of the system. In this particular example, the coupling was triggered due to the mode frequencies being very close. Another possible trigger can be disturbances entering the system at frequencies close to the system modes, i.e. fluctuating RES, which, to the best of authors' knowledge, hasn't been studied yet.

\section{B. Mode Coupling Triggered by Fluctuating RES}

Unlike the conventional energy sources, some RES can exhibit fluctuating behaviour due to the variability of the primary energy source. While geothermal, tidal and solar thermal cannot change the output quickly due to the large inherent inertia, solar PV, wave and wind can. Solar PV doesn't possess any energy buffer so the output can fluctuate due to varying cloud coverage. The resulting fluctuations however will likely be aperiodic. Wave and wind generation, on the other hand, can generate periodic power fluctuations. Unlike wind, wave generation is still in its infancy, so little operational data is available in the literature. In this paper, we therefore consider wind power as a trigger of possible mode coupling.

The fluctuation in the wind can be thought of as resulting from a composite of sinusoidally varying winds superimposed on the mean steady wind [14]. If the frequency of a sufficient strong wind component matches the frequency of one the system's poorly damped oscillatory modes, the mode gets excited by the fluctuating RES, which results in resonance. This phenomenon is called mode coupling and is not restricted by the electrical distance between the source and the machine participating in the affected mode. In other words, mode coupling can affect both local and inter-area modes.

\section{Wind Power Spectrum}

The variability of wind spans multiple time scales. Mediumand long-term fluctuations can be modelled with Van der Hoven's spectral model, while for short-term fluctuations, capturing the turbulent behaviour, either von Karman's or Kaimal spectral models are typically used [15]. Van der Hoven's spectrum is modelled as a stationary random process, whereas the turbulence spectrums, either von Karman's or Kaimal's, are non-stationary. Van der Hoven's spectrum spans the range between $0.001 \mathrm{cycles} / \mathrm{h}$ (inter-seasonal variations) and 1000 cycles/h (intra-minute variations). The turbulent short-term power spectrum spans the frequencies between 0.01 and $4 \mathrm{~Hz}$, which covers the typical frequency range of the local and inter-area oscillatory modes (between 0.25 and $2 \mathrm{~Hz}$ ). The turbulent wind fluctuations should therefore be considered in the analysis of mode coupling due to fluctuation RES. For illustration, a sample Van Der Hoven's and Kaimal wind power spectra are shown in Figs. 1 and 2, respectively.

Observe in Fig. 2 the effect of the rotational sampling of the wind turbine blades caused by the tower shadow. For the most common 3-blade turbine, the sharp spike occurs at the triple of the turbine's rotational speed. The exact frequency thus depends on the rotational speed of the turbine, determined by the design and the control method used. This deserves further attention, as the frequency of the spike could be close 


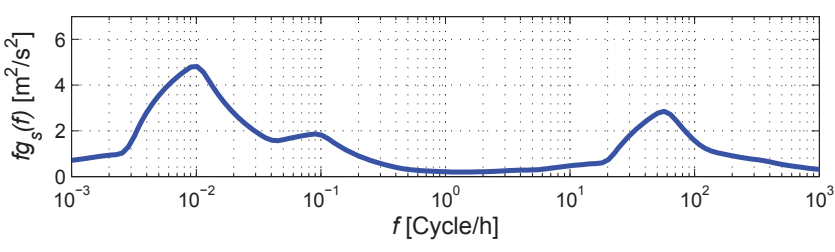

Fig. 1. Van Der Hoven's wind power spectrum.

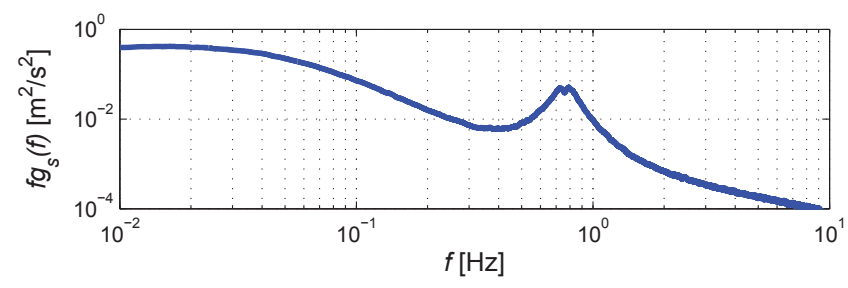

Fig. 2. Kaimal's wind power spectrum.

to the frequency of an oscillatory mode. This particular issue is however beyond the scope of the paper.

\section{Identifying the State Space Model of the SYSTEM}

Power systems are time varying and highly non-linear so it is almost impossible to identify the non-linear models. Instead, linearization could be used to analyse the impact of a small deviation in system's inputs. DIgSILENT/PowerFactory doesn't allow the user to obtain the linearized state-space model of the system. Instead, we used the N4SID sub-space system identification technique available in Matlab's System Identification Toolbox [16]. The state-space model has been used to obtain the transfer functions of interest for a particular operating condition, as described later.

One of the most important aspects of system identification is the selection of the probing signal [17]. Firstly, the frequency spectrum needs to cover the frequency band of interest, and, secondly, the amplitude of the signal must be sufficiently high to excite the critical oscillatory modes without pushing the system into a nonlinear zone [18]. We tested the following probing signals: Random Gaussian Signal, Random Binary Signal, Pseudo Random Binary Signal (PRBS), and Sum of Sinusoid Signal. PRBS was chosen due to its superior performance over the widest frequency range.

Intuitively, perturbation of the system with a fluctuating RES is similar to system identification. In response to the perturbation signal with a wide frequency spectrum, most of the frequency band is filtered out except for the oscillatory frequency, where the system resonates with the perturbation signal.

\section{A. Numerical Subspace State Space System Identification}

In our study, system identification was used to identify the transfer functions between the wind turbine, modeled as a controlled current using the in-built converter model, as the input and the angular speeds of the four generators as the outputs to study the resonant behaviour, i.e. the mode coupling between the fluctuating RES and the existing oscillatory modes. The resulting system identification problem is thus Single Input Multiple Output (SIMO). The model with $m$ inputs, $p$ outputs and $n$ states was estimated in discrete domain and the converted into continuous domain resulting in

$$
\begin{aligned}
& \dot{\mathbf{x}}(t)=\mathbf{A x}(t)+\mathbf{B u}(t)+\mathbf{K e}(t) \\
& \mathbf{y}(t)=\mathbf{C x}(t)+\mathbf{D} \mathbf{u}(t)+\mathbf{e}(t)
\end{aligned}
$$

where $\mathbf{x} \in \mathbb{R}^{n}$ is the state vector; $\mathbf{u} \in \mathbb{R}^{m}$ is the input vector; $\mathbf{y} \in \mathbb{R}^{p}$ denotes the output vector; and $\mathbf{A} \in \mathbb{R}^{n \times n}, \mathbf{B} \in$ $\mathbb{R}^{n \times m}, \mathbf{C} \in \mathbb{R}^{p \times n}$ and $\mathbf{D} \in \mathbb{R}^{p \times m}$ are the system matrices. $\operatorname{Ke}(t) \in \mathbb{R}^{n}$ and $\mathbf{e}(t) \in \mathbb{R}^{p}$ are, respectively, the disturbances and the noise acting on the system. In our case $m=1, p=4$ and $n=13$. The dynamic order of the original system is 49 .

\section{TEST System}

\section{A. Test Bed: Two-area System}

We used the four-machine, two-area test system proposed in [19] as the test bed. The system consists of four generators located in two areas; G1 and G2 in the east, and G3 and G4 in the west. A wind power plant, modelled as a single 120MW Type IV (synchronous machine with a fully rated converter) wind turbine has been connected to the high voltage bus near G2. The system is shown in Fig. 3. As a result, the power of the slack bus (G1) has been reduced to keep the system in balance.

Three oscillatory modes exist in this system: one interarea mode with the generators in the east oscillating against the generators in the west; and two local modes, one between G1 and G2 and the other between G3 and G4. We considered the case of a thyristor exciter with high transient gain and PSS. To get mode coupling in the system, the renewable generator (sender) needs to have a component with a frequency similar to one of the existing oscillatory modes. To this end, we modified some PSS parameters to create two cases with a different oscillatory behaviour-see Table I.

Other parameters were left unchanged, resulting in three study cases: (1) the original case, (2) a case where a local mode (G3, G4) with reduced damping resonates with the wind turbine; and (3) a case with an unstable local mode (G3, G4). In all three cases, we first identified the sub-space linearized

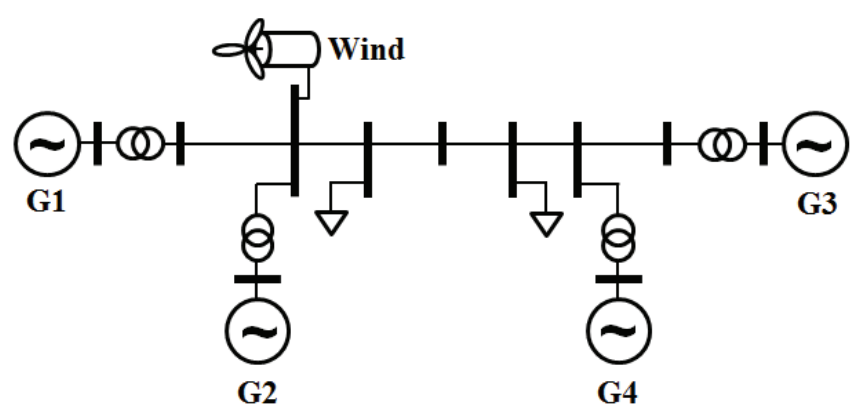

Fig. 3. Two Area Test System. 
TABLE I

MODIFIED EXCITER AND PSS PARAMETERS.

\begin{tabular}{ccccccc}
\hline & & G1 & & \multicolumn{3}{c}{ G1 } \\
\hline Case & $\mathbf{1}$ & $\mathbf{2}$ & $\mathbf{3}$ & $\mathbf{1}$ & $\mathbf{2}$ & $\mathbf{3}$ \\
\hline$K_{\text {stab }}$ & 20 & 200 & 200 & 20 & 20 & 20 \\
\hline$T_{2}$ & 0.02 & 0.02 & 0.02 & 0.02 & 0.02 & 0.02 \\
\hline$T_{3}$ & 3 & 30 & 30 & 3 & 30 & 30 \\
\hline \hline & & $\mathbf{G 3}$ & & & $\mathbf{G 4}$ & \\
\hline Case & $\mathbf{1}$ & $\mathbf{2}$ & $\mathbf{3}$ & $\mathbf{1}$ & $\mathbf{2}$ & $\mathbf{3}$ \\
\hline$K_{\text {stab }}$ & 20 & 185 & 200 & 20 & 20 & 5 \\
\hline$T_{2}$ & 0.02 & 0.17 & 0.17 & 0.02 & 0.02 & 0.02 \\
\hline$T_{3}$ & 3 & 3 & 3 & 3 & 3 & 5 \\
\hline \hline
\end{tabular}

models of the system that were used to obtain the transfer functions between the wind turbine as the input and the angular speeds of all four generators as the outputs. This enabled us to identify possible resonance in the system subject to fluctuations in the wind power. Next, we injected a sinusoidal signal at three different frequencies, representing fluctuating wind power spectral components, and simulated the response of the system in time domain.

\section{B. Case 1: Standard System}

The standard system corresponds to the case with the thyristor exciter with high transient gain and PSS in [19]. Fig. 4 shows the oscillatory modes in the complex plane. Observe that the local modes $(\mathrm{G} 1, \mathrm{G} 2)$ and $(\mathrm{G} 3, \mathrm{G} 4)$ have very similar frequencies.

Fig. 5 shows the Bode plots of the transfer functions between the wind power and the machine angular speeds of the four generators.

Three signals with different frequency are injected: (A) interarea mode frequency; (B) local mode frequency; and (C) a signal with a frequency significantly different from mode frequencies. The spike in the bode plot around frequency

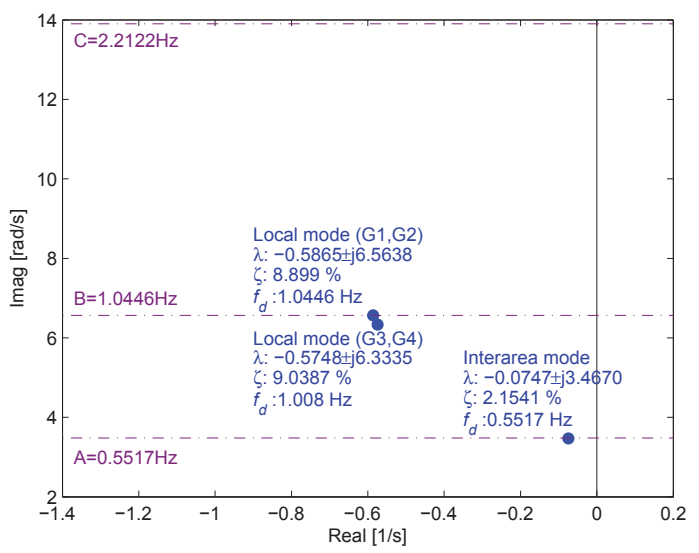

Fig. 4. Case 1: Oscillatory modes and the frequencies of the injected signals.

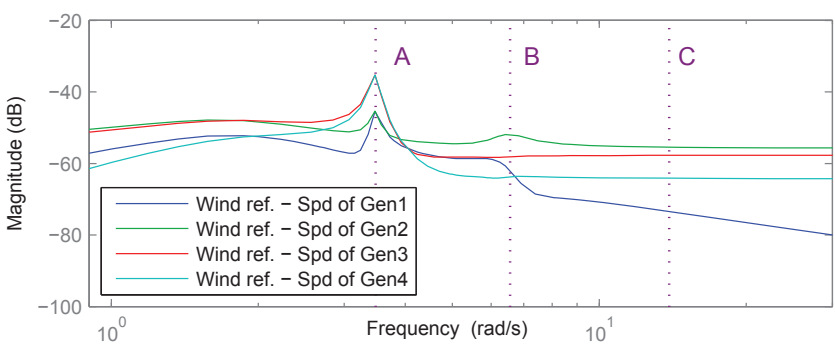

Fig. 5. Case 1: Oscillatory modes and the frequencies of the injected signals.
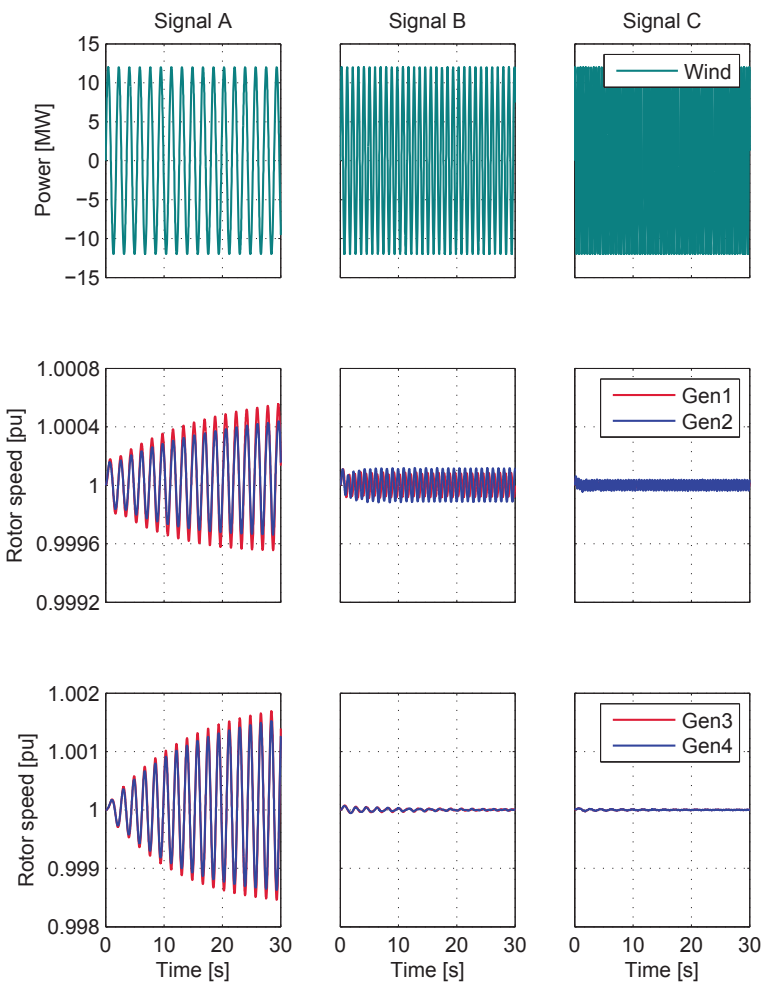

Fig. 6. Case 1: Time-domain simulation results.

A indicates that the injected sinusoidal signal might interact with the interarea mode while we don't expect any interaction for frequencies B and C. This is confirmed by time-domain simulations, Fig. 6. Observe in Fig. 6 the amplified response of the four generators subject to a sinusoidal wind power injection with frequency A. As expected, frequencies B and C have negligible impact.

\section{Case 2: Resonant Local Mode}

In this case, we modified the parameters of the excitation system to reduce damping of the local mode $(\mathrm{G} 3, \mathrm{G} 4)$ ). Fig. 7 and Fig. 8 show the oscillatory modes in the complex plane and the Bode plots, respectively.

Unlike in Case 1, we now expect the wind turbine input to resonate with the three modes for frequencies $\mathrm{A}$ and $\mathrm{C}$. In addition, because the frequencies $\mathrm{A}$ and $\mathrm{C}$ are similar, we also expect frequency $\mathrm{B}$, which lies between $\mathrm{A}$ and $\mathrm{C}$, to cause 


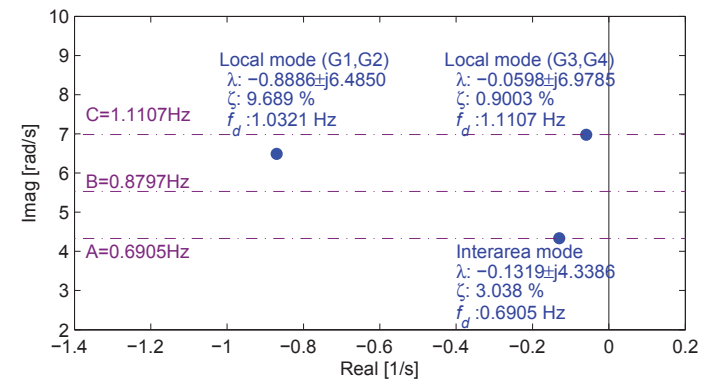

Fig. 7. Case 2: Oscillatory modes and the frequencies of the injected signals.

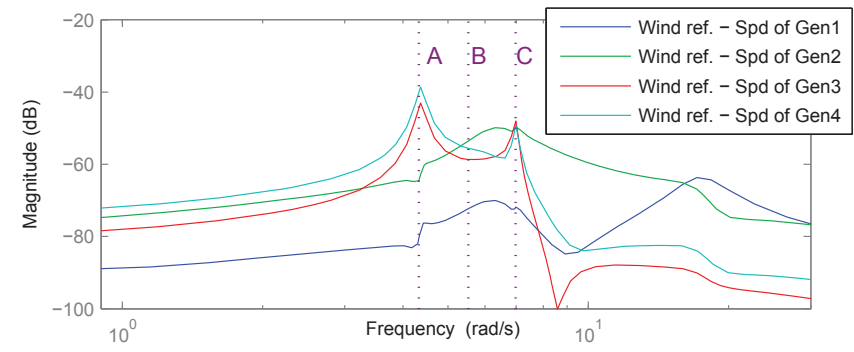

Fig. 8. Case 2: Bode plots.

resonance. Fig. 9 shows the simulation results for the three cases.

As expected, all three signals cause resonance, with frequency A having the largest impact. We can see in Fig. 9 that Signal B, whose frequency lies between the frequencies of the interarea mode and the local mode (G3 and G4), causes slight oscillation of the angular speed of the generators but not significant amplification. The response of G3 and G4 indicates that the injection of a sinusoidal disturbance in one part of the system causes resonance in a distant part of the system, which confirms our assumption and also agrees with what has been observed in the WECC system [12].

\section{Case 3: Unstable Local Mode}

In the last case we destabilised the local mode $(\mathrm{G} 3, \mathrm{G} 4)$ to study the impact of fluctuating RES on a poorly damped system. Fig. 10 and Fig. 11 show the oscillatory modes in the complex plane and the Bode plots, respectively. In this case, only one frequency has been chosen, matching the frequency of the poorly damped mode $(\mathrm{G} 3, \mathrm{G} 4)$.

Figs. 12 and 13 show the results of time-domain simulation. Observe that, unlike in the previous two cases, the injected signal is now damped. In the first 10 seconds after the disturbance the system seems to have stabilized. However, a longer time-frame of 200 seconds shows that the oscillations grow in amplitude, which indicates unstable behavior. This indicates that even a small perturbation at a particular frequency can excite a poorly damped oscillatory mode.

\section{CONClusion}

The existing research has so far taken a "quasi-static" approach in stability analysis of future grid scenarios, by
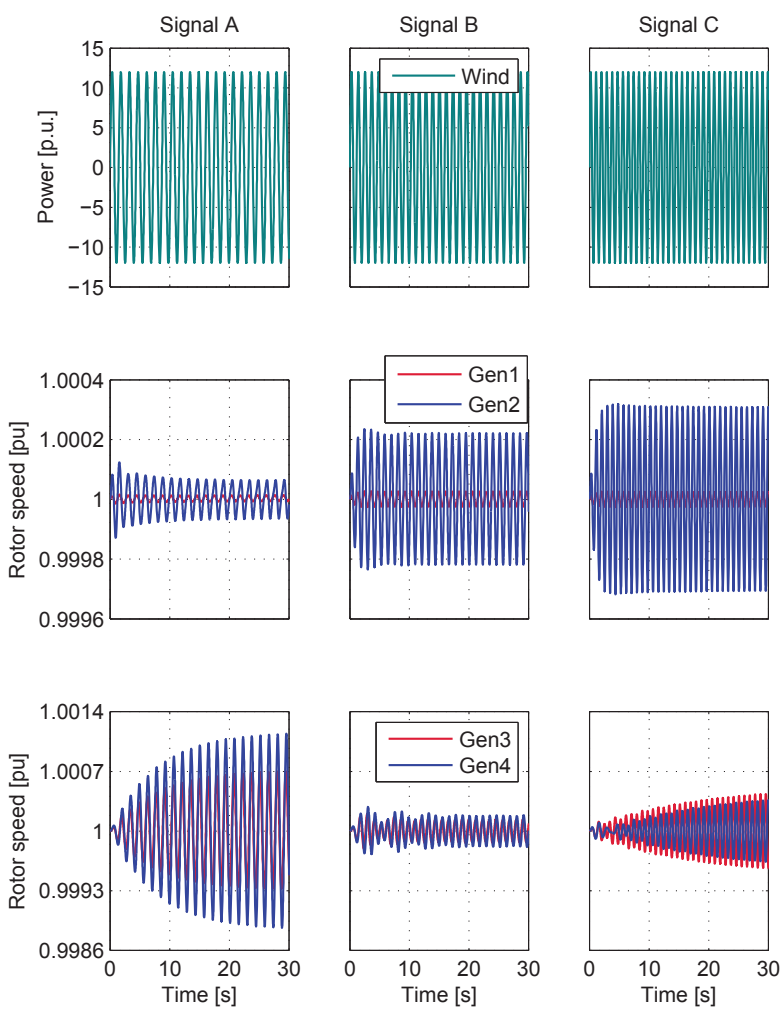

Fig. 9. Case 2: Time-domain simulation results.

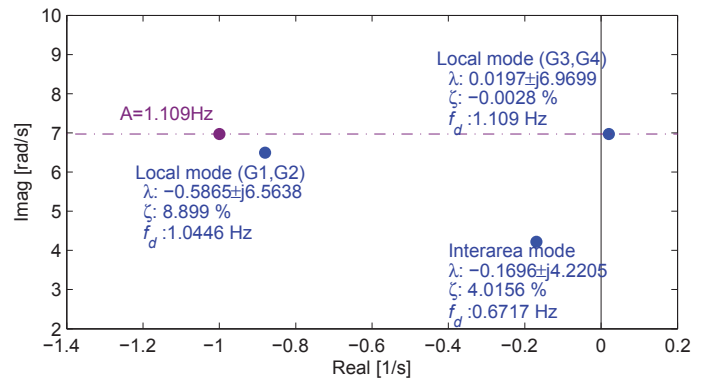

Fig. 10. Case 3: Oscillatory modes and the frequency of the injected signal.

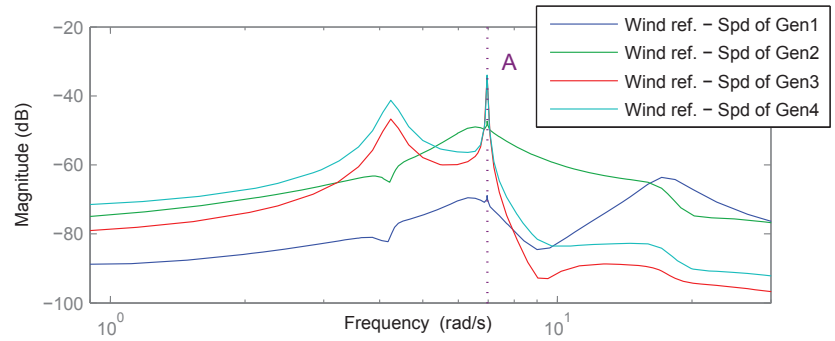

Fig. 11. Case 3: Bode plots. 

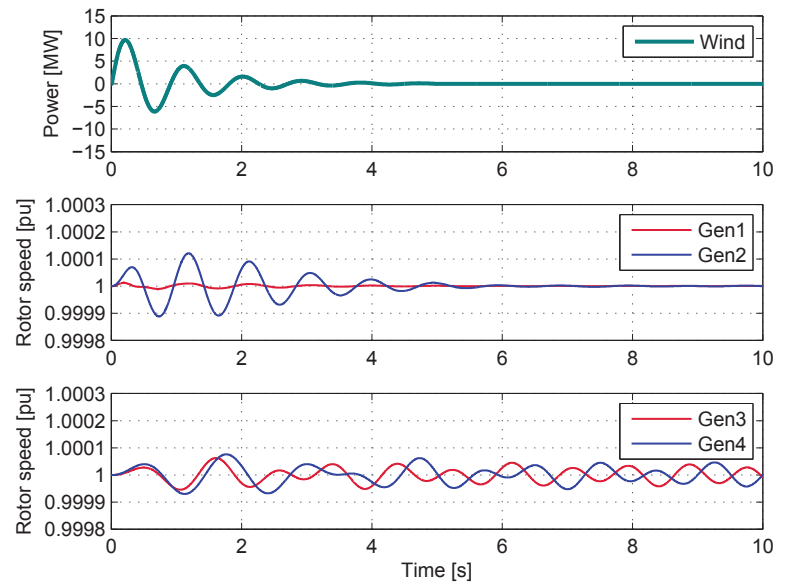

Fig. 12. Case 3: Time-domain simulation results; short-term evolution.
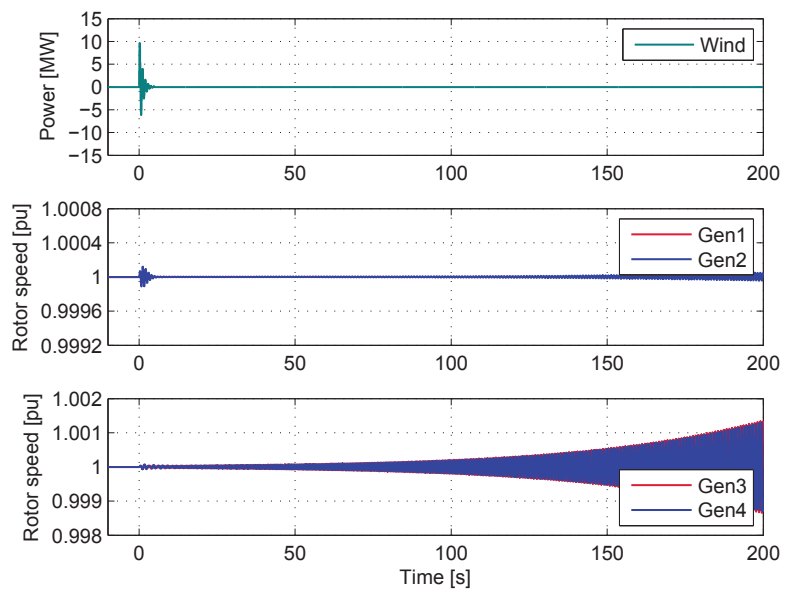

Fig. 13. Case 3: Time-domain simulation results; long-term evolution.

assuming that power is provided from static generation units. The focus has thus been limited to how much damping ratio and frequency changes as a result of changing RES penetration. However, it appears that dynamic effects of fast variations in the output of RES should be considered as well.

This paper has made an attempt to cover this gap by studying the impact of fluctuating wind power on oscillatory stability of a power system. Wind power can have spectral components similar in frequency to oscillatory power system modes. The motivation for the research was thus to analyse possible modal interaction, which can lead to resonance that can negatively affect power system stability. The study cases analysed in the paper confirmed our expectations. We found that fluctuating wind power can indeed amplify power system oscillations, even when the machines participating in the affected modes were located electrically far away from the wind turbine. Due to the increasing penetration of wind power worldwide, the problem of possible modal interaction between RES and the existing power system oscillatory modes certainly deserves more attention.

We hope that the research presented in the paper will help the transition to future grids predominantly relying on renewable energy sources.

\section{ACKNOWLEDGMENT}

This research was partly supported by Basic Science Research Program through the National Research Foundation of Korea (NRF) funded by the Ministry of Education, Science, Technology (No. NRF- 2012K2A1A2033153).

\section{REFERENCES}

[1] The University of Melbourne Energy Research Institute, "Zero Carbon Australia Stationary Energy Plan,” Tech. Rep., 2010.

[2] Australian Energy Market Operator (AEMO), "100 Per Cent Renewables Study-Modelling Outcomes," Tech. Rep. July, 2013.

[3] J. Slootweg and W. Kling, "The impact of large scale wind power generation on power system oscillations," Electric Power Systems Research, vol. 67 , no. 1 , pp. 9-20, Oct. 2003.

[4] G. Tsourakis, B. Nomikos, and C. Vournas, "Contribution of Doubly Fed Wind Generators to Oscillation Damping," IEEE Transactions on Energy Conversion, vol. 24, no. 3, pp. 783-791, Sep. 2009.

[5] J. Sanchez-Gasca, N. Miller, and W. Price, "A modal analysis of a twoarea system with significant wind power penetration," in IEEE PES Power Systems Conference and Exposition, 2004. IEEE, 2004, pp. $1537-1541$.

[6] S. Eftekharnejad, V. Vittal, G. T. Heydt, B. Keel, and J. Loehr, "Small Signal Stability Assessment of Power Systems With Increased Penetration of Photovoltaic Generation: A Case Study," IEEE Transactions on Sustainable Energy, vol. 4, no. 4, pp. 960-967, Oct. 2013.

[7] D. Gautam, V. Vittal, and T. Harbour, "Impact of Increased Penetration of DFIG-Based Wind Turbine Generators on Transient and Small Signal Stability of Power Systems," IEEE Transactions on Power Systems, vol. 24, no. 3, pp. 1426-1434, Aug. 2009.

[8] B. Tamimi, C. Canizares, and K. Bhattacharya, "System Stability Impact of Large-Scale and Distributed Solar Photovoltaic Generation: The Case of Ontario, Canada," IEEE Transactions on Sustainable Energy, vol. 4, no. 3, pp. 680-688, Jul. 2013.

[9] CIGRE, "Grid Integration of Wind Generation," Working Group 08 of Study Committee C6, Tech. Rep., 2009.

[10] Australian Energy Market Operator (AEMO), "Integrating Renewable Energ-Wind Integration Studies Report," Tech. Rep., 2013.

[11] D. K. Mugwanya and J. E. Van Ness, "Mode Coupling in Power Systems," IEEE Transactions on Power Systems, vol. 2, no. 2, pp. 264 269, 1987.

[12] M. Klein, G. Rogers, S. Moorty, and P. Kundur, "Analytical investigation of factors influencing power system stabilizers performance," IEEE Transactions on Energy Conversion, vol. 7, no. 3, pp. 382-390, 1992.

[13] P. Kundur, J. Paserba, V. Ajjarapu, G. Andersson, A. Bose, C. Canizares, N. Hatziargyriou, D. Hill, A. Stankovic, C. Taylor, T. Van Cutsem, and V. Vittal, "Definition and classification of power system stability ieee/cigre joint task force on stability terms and definitions," Power Systems, IEEE Transactions on, vol. 19, no. 3, pp. 1387-1401, Aug 2004.

[14] J. F. Manwell, J. G. McGowan, and A. L. Rogers, Wind Energy Explained: Theory, Design and Application. Wiley, 2010.

[15] T. Burton, N. Jenkins, D. Sharpe, and E. Bossanyi, Wind Energy Handbook. John Wiley \& Sons, 2011.

[16] P. Van Overschee and B. De Moor, Subspace identification for linear systems: theory, implementation, applications, Volume 1. Kluwer Academic Publishers, 1996.

[17] Y. Pipelzadeh, N. Chaudhuri, B. Chaudhuri, and T. Green, "System stability improvement through optimal control allocation in voltage source converter-based high-voltage direct current links," Generation, Transmission Distribution, IET, vol. 6, no. 9, pp. 811-821, September 2012.

[18] J. Hauer, W. Mittelstadt, K. Martin, J. Burns, H. Lee, J. Pierre, and D. Trudnowski, "Use of the wecc wams in wide-area probing tests for validation of system performance and modeling," Power Systems, IEEE Transactions on, vol. 24, no. 1, pp. 250-257, Feb 2009.

[19] P. Kundur, Power System Stability and Control. McGraw-Hill, 1994. 